\title{
Multi-user MIMO CDMA systems using complete complementary sequences
}

\author{
C. Khirallah, P. Coulton, H. Rashvand and N. Zein
}

\begin{abstract}
The authors propose a novel use of complete complementary (CC) sequences for increased spectral efficiency in a multiple-input-multiple-output (MIMO) code division multiple access (CDMA) system. The new method overcomes many of the problems and limitations persistent in single-input-single-output (SISO) and proves that under the proposed MIMO CCCDMA system, the capacity for the number of supported users increases linearly by the number of transmitters. The paper also shows that the MIMO CC-CDMA system demonstrates a superior performance over those using traditional Walsh spreading sequences (Walsh-CDMA). Results include the bit error rate comparison for CC-CDMA frequency selective fading channels and that of the Walsh-CDMA under flat fading channels.
\end{abstract}

\section{Introduction}

The new paradigm of multiple-input-multiple-output (MIMO) is a promising approach that can lead to significant bandwidth efficiency. The high spectral efficiency can be achieved by transmitting different signals on different transmit antennas simultaneously using the same frequency channel in conjunction with multiple receive antennas under an appropriate receive scheme [1, 2].

The spectral efficiency can be exploited in MIMO systems depending on a number of phenomena, including received power, noise, and co-channel interference. However, the multi-dimensional statistical behaviour of the MIMO fading channel is most significant to the system performance and also supports spatial multiplexing (SM) [3]. When used in conjunction with the vertical Bell Labs layered space time (VBLAST) that uses the same spreading sequence to transmit independent data streams from different antennas termed 'sequence re-use', the data rate increases, under narrowband and flat fading channel conditions $M$ times, where $M$ is the number of transmit antennas [4, 5].

However, using the code division multiple access (CDMA) with the commonly used Walsh sequences and VBLAST multi-user detector (MUD) suffers from high level multiple access interference (MAI) and significant inter-symbol interference (ISI) in wideband and frequencyselective fading channels resulting in severe degradation in system performance [6]. This is due to the corrupted initial orthogonality between Walsh sequences at the de-spread stage at the receiver. Many solutions have been proposed to

(C) The Institution of Engineering and Technology 2006

IEE Proceedings online no. 20050404

doi:10.1049/ip-com:20050404

Paper first received 31st July 2005 and in revised form 27th January 2006

C. Khirallah and P. Coulton are with the Department of Communication Systems, University of Lancaster, B Floor, INFOLAB 21, South Drive, Lancaster LA1 4WA, UK

H. Rashvand is with the School of Engineering, University of Warwick, Coventry, CV4 7AL, UK

N. Zein is with the Telecommunications Consulting Department, NEC Europe Ltd, SL1 3UA, UK

E-mail: c.khriallah@lancaster.ac.uk overcome this problem resulting in higher complexity. Some of these include:

(i) linear-chip equalisation combined with multiple nonlinear parallel interference cancellation (IC) [7];

(ii) CDMA combined with orthogonal frequency division multiplexing (OFDM), which is capable of delivering high data rates in multipath environment by largely eliminating the effects of ISI [8]. However, owing to inter-carrier interference (ICI) in OFDM mobile communication systems, the bit error rate (BER) of the received signals is extremely sensitive to Doppler frequency shifts and carrier synchronisation errors [9];

(iii) Using alternative spreading sequences that exhibit resilience towards multipath time-dispersive effects. To this end, $\mathrm{CC}$ sequences have been considered [10] as a candidate for multi-carrier CDMA, offering much higher spreading efficiency (SE), a MAI-free operation in both down-link (synchronous channel) and up-link (asynchronous) and the ability to support multi-rate service with a low complexity rate-matching algorithm. This however only applies to singleinput-single-output (SISO) systems with very few supported users. This is due to a restriction imposed on the length of the $\mathrm{CC}$ element sequence $\left(K_{C C}\right)$ by manageable signal levels in the required multilevel $\left(2 \times K_{C C}+1\right)$ digital modem.

This paper presents a new MIMO CC-CDMA system to overcome the aforementioned major limitations. Our MIMO CC-CDMA system applies the SM technique but instead of repeating a single sequence on different antennas its sets of $\mathrm{CC}$ sequences are reused on different transmit antennas to spread independent data streams. This technique, called 'set re-use', has the following benefits: (i) increasing the maximum number of supported users, which is no longer limited by the manageable signal levels in the digital modem, as shown in Table 1; (ii) by reducing the length of the $\mathrm{CC}$ element sequence and using multiple antennas in the MIMO CC-CDMA, it may be also possible to reduce the peak-to-average power ratio (PAPR), and hence, the increased power efficiency of the power amplifier, meaning that the average radiated power is reduced in order to avoid the nonlinear distortion of transmitted signal [11].

Table 1 shows how the proposed MIMO CC-CDMA enables us to increase the number of supported users, while 
Table 1: Ability of the proposed MIMO CC-CDMA to either increase number of users or reduce the complexity of the required multilevel digital modem

\begin{tabular}{llll}
\hline $\begin{array}{l}\text { Element } \\
\text { sequence } \\
\text { length }\left(K_{C C}\right)\end{array}$ & $\begin{array}{l}\text { Number } \\
\text { of users } \\
\left(M \sqrt{K_{C C}}\right)\end{array}$ & $\begin{array}{l}\text { Antenna } \\
\text { structure } \\
(M, P)\end{array}$ & $\begin{array}{l}\text { Signal multilevel } \\
\text { in digital modem } \\
\left(2 K_{C C}+1\right)\end{array}$ \\
\hline 16 & 4 & $(1,1)$ & 33 \\
16 & 8 & $(2,2)$ & 33 \\
4 & 4 & $(2,2)$ & 9 \\
\hline
\end{tabular}

maintaining the signal level, or equally to reduce the required modem complexity while maintaining the same number of users.

The rest of this paper is organised as follows: Section 2 presents, firstly, the generation of CC sequences, and then explains the ideal correlation properties of $\mathrm{CC}$ sequences compared to those of Walsh and Gold sequences. This comparison provides an indication of the likely system interference and forms the basic criteria for the selection of CC spreading sequences for wideband MIMO CDMA mobile systems. Section 3 presents the general model of the proposed MIMO CC-CDMA system and followed by a detailed example of 2transmit-2receive $(2,2)$ CC-CDMA system. This includes a detailed description of how MAI, ISI and spatial interference (SPI) cancellation is achieved. Section 4 provides the simulation parameters for two comparable CC-CDMA and Walsh-CDMA simulation models, and the simulated BER performance. Finally conclusions are presented in Section 5.

\section{Complete complementary sequences sets}

The origin of CC sequences can be traced back to the 1960s, when Turyn [12] and Taki et al. [13] described a class of binary sequences, whose elements are either -1 or +1 , and whose auto-correlation function is zero for all even (2-mulitple) shifts except the zero shift. These sequences have been called even-shift orthogonal sequences. Suehiro [14] extended the concept to the generation of CC sequence families/sets whose auto-correlation function is zero for all even and odd shifts except the zero shift and whose crosscorrelation function, for any pair, is zero for all possible shifts. According to [14] in order for $N$ sets, each made up of $N$ sequences, to be $\mathrm{CC}$ sequences of order $N$ they must satisfy: (i) the sum of the $N$ auto-correlation functions, for each set, is zero for any shift, except the zero shift (this set is called 'auto-complementary sequence of order $N$ '); (ii) the sum of the $N$ cross-correlation functions, between two sets of $N$ sequences, is zero for any shift (each of these two sets is called 'cross-complementary sequence of order $N$ ').

\subsection{Generating CC sequences of order N using cross-orthogonal sequences of length $K_{C C}=N^{2}$}

$\mathrm{CC}$ sequences can be constructed using Hadamard matrices [5], such as an $N$-dimensional orthogonal matrix $\boldsymbol{A}_{N}$, which is generated recursively using the Kronecker product of a core matrix $\boldsymbol{A}_{2}(2 \times 2)$ and $\boldsymbol{A}_{n / 2}$ matrix as shown in (1) and (2).

$$
\begin{gathered}
\boldsymbol{A}_{N}=\boldsymbol{A}_{2} \otimes \boldsymbol{A}_{N / 2} \\
\boldsymbol{A}_{N}=\left[\begin{array}{ll}
\boldsymbol{A}_{N / 2} & \boldsymbol{A}_{N / 2} \\
\boldsymbol{A}_{N / 2} & \overline{\boldsymbol{A}}_{N / 2}
\end{array}\right]
\end{gathered}
$$

where $\boldsymbol{A}_{1}$ is positive waveform $(+1), \overline{\boldsymbol{A}}_{N / 2}$ is the complement of $\boldsymbol{A}_{N / 2}, N=2^{k}$ and $k=1,2, \ldots, \log _{2} N$.
The following steps are used to generate $\mathrm{CC}$ sequences of length $N^{2}$ :

- Constructing an auto-orthogonal sequence of length $N^{2}$. Let $\boldsymbol{D}$ be a sequence of length $N^{2}$ that is constructed by linking all rows of a Hadamard matrix $\boldsymbol{A}_{N}(N \times N)$, which is given in (2).

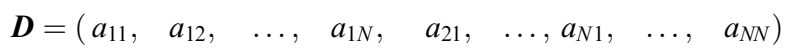

Since $\boldsymbol{A}_{N}(N \times N)$ is orthogonal matrix, the auto-correlation function of $\boldsymbol{D}$ is zero for all $N$-multiple shifts except the zero shift.

- Constructing a set of $N$ cross-orthogonal sequences of length $N^{2}$. Let $[\boldsymbol{A}]_{1},[\boldsymbol{A}]_{2}, \ldots,[\boldsymbol{A}]_{N}$ be the rows of the Hadamard matrix $\boldsymbol{A}_{N}(N \times N)$ and now let us make sequences of length $N^{2}$ as the rows of orthogonal matrix $\boldsymbol{B}$ given by

$$
\boldsymbol{B}=\left[\begin{array}{c}
\boldsymbol{B}_{1} \\
\boldsymbol{B}_{2} \\
\vdots \\
\boldsymbol{B}_{N}
\end{array}\right]=\left[\begin{array}{rrrr}
a_{11}[\boldsymbol{A}]_{1}, & a_{12}[\boldsymbol{A}]_{2}, & \ldots, & a_{1 N}[\boldsymbol{A}]_{N} \\
a_{21}[\boldsymbol{A}]_{1}, & a_{22}[\boldsymbol{A}]_{2}, & \ldots, & a_{2 N}[\boldsymbol{A}]_{N} \\
& \vdots & & \\
a_{N 1}[\boldsymbol{A}]_{1}, & a_{N 2}[\boldsymbol{A}]_{2}, & \ldots, & a_{N N}[\boldsymbol{A}]_{N}
\end{array}\right]
$$

It can be shown that since $\boldsymbol{B}$ and $\boldsymbol{A}$ are orthogonal matrices, the set of $\boldsymbol{B}_{1}, \boldsymbol{B}_{2}, \ldots, \boldsymbol{B}_{N}$ are both auto-orthogonal and cross-orthogonal sequences of length $N^{2}$.

\subsection{Constructing $N$ sets of $C C$ sequences of order $N$ using auto-orthogonal sequences of length $N^{2}$}

Using sequences in (4) and a Hadamard matrix $\boldsymbol{A}_{N}(N \times N)$, we can construct $N$ sequences of length $N^{2}$ as shown in (5).

$$
\begin{aligned}
& w_{i j}=\left(b_{i 1} a_{j 1}, \quad b_{i 2} a_{j 2}, \quad \ldots, \quad b_{i N} a_{j N},\right. \\
& b_{i(1+N)} a_{j 1}, \quad b_{i(2+N)} a_{j 2}, \quad \ldots, \quad b_{i(2 N)} a_{j N}, \\
& \left.b_{i\left(N^{2}-(1+N)\right)} a_{j 1}, \quad b_{i\left(N^{2}-(2+N)\right.} a_{j 2}, \quad \ldots, \quad b_{i N^{2}} a_{j N}\right)
\end{aligned}
$$

where $j$ and $i=1,2, \ldots, N$. From (5) each set of sequences $\left\{w_{i 1}, \ldots, w_{i N}\right\}$ forms an auto-complementary sequence of order $N$, and any two of the generated $N$ sets $\left\{w_{11}, \ldots, w_{1 N}\right\}, \ldots,\left\{w_{i 1}, \ldots, w_{i N}\right\},\left\{w_{N 1}, \ldots, w_{N N}\right\}$ satisfy the property of the cross-complementary sequence of order $N$, where $w_{i j}$ is a CC element sequence of length $N^{2}$

Table 2 shows a selected number of the eight sets of $\mathrm{CC}$ sequences (the first two sequences of each set), for a set size 8 and sequence length 64 . These sequences are generated using an $8 \times 8$ Hadamard matrix given in (6).

$$
A_{8}=\left[\begin{array}{cc}
++++ & ++++ \\
+-+- & +-+- \\
++-- & ++-- \\
+--+ & +--+ \\
++++ & ---- \\
+-+- & -+-+ \\
++-- & --++ \\
+--+ & -++-
\end{array}\right]
$$


Table 2: A selected number of $C C$ sequences, for an element sequence of length 64 , generated using an $8 \times 8$ Hadamard matrix

\begin{tabular}{|c|c|}
\hline CC sequences/sets & $\mathrm{CC}$ orthogonal element sequences \\
\hline Set 1/user 1 & $\begin{aligned} w_{1}= & (+++++++++-+-+-+-++--++--+--++--+ \\
& ++++----+-+--+-+++----+++--+-++-) \\
w_{2}= & (+-+-+-+-+++++++++--++--+++--++-- \\
& +-+--+-+++++----+--+-++-++----++)\end{aligned}$ \\
\hline Set 2/user 2 & $\begin{aligned} w_{9}= & (++++++++-+-+-+-+++--++---++--++- \\
& ++++-----+-++-+-++----++-++-+--+) \\
w_{10}= & (+-+-+-+---------+--++--+--++--++ \\
& +-+--+-+----+++++--+-++---++++--)\end{aligned}$ \\
\hline Set 8/user 8 & $\begin{aligned} w_{57}= & (++++++++-+-+-+-+--++--+++--++--+ \\
& ----+++++-+--+-+++----++-++-+--+) \\
w_{58}= & (+-+-+-+----------++--++-++--++-- \\
& -+-++-+-++++----+--+-++---++++--)\end{aligned}$ \\
\hline
\end{tabular}

\subsection{CC sequences correlation properties}

$\mathrm{CC}$ sequences exhibit the following fundamental differences compared to traditional CDMA spreading sequences such as (Gold sequences, m-sequences, Walsh Hadamard sequences, etc):

- The mutual orthogonality of CC sequences is observed between 'sets' (sets of element sequences) instead of between single element sequences as for other spreading sequences such as Gold and Walsh sequences. In other words, for the proposed CC-CDMA system every user will be assigned one set of sequences, where the total number of available sets is defined as

$$
F=N=\sqrt{K_{C C}}=2^{j}
$$

where, $j=1,2, \ldots$ is a positive integer.

- $\mathrm{CC}$ sequences exhibit unique correlation properties with zero cross correlation and zero out-of-phase autocorrelation are ensured for any relative shifts between two sequences. The auto-correlation function $\left(\psi_{X X}(i)\right)$ of a set $\mathrm{X}$ of $\mathrm{CC}$ sequences is defined, in (8), as the sum of the auto-correlation functions for all element sequences in $\mathrm{X}$

$$
\psi_{\boldsymbol{X X}}(i)=\left\{\begin{array}{l}
\sum_{n=1}^{N_{C C}} \sum_{k=1}^{K_{C C}-i} w_{n}(k) w_{n}(k+i)^{*}=0, \quad 1 \leq i \leq K_{C C}-1 \\
\sum_{n=1}^{N_{C C}} \sum_{k=1}^{K_{C C}+i} w_{n}(k) w_{n}(k-i)^{*}=0, \quad 1-K_{C C} \leq i \leq-1 \\
\sum_{n=1}^{N_{C C}} \sum_{k=1}^{K_{C C}} w_{n}(k) w_{n}(k)^{*}=K_{C C} \times N_{C C}, \quad i=0
\end{array}\right.
$$

$n=1,2, \ldots, N_{C C}\left(=\sqrt{K_{C C}}\right)$ is the number of element sequences per set. $k=1,2, \ldots, K_{C C}$ and $i=0, \pm 1$, $\pm 2, \ldots, \pm\left(K_{C C}-1\right)$ is an integer that represents the number of cyclic shifts (given by chip-period $\tau_{C}$ ) for each element sequence, and $w_{n}(k)$ is the $k$ th chip of the $n$th element sequence. From (8), it can be seen that $\psi_{\boldsymbol{X} \boldsymbol{X}}(i)$ equals zero for all time shifts except the zero-shift, thus indicating the possibility of reduced level of ISI in multipath fading channels.

For CC sequences the cross-correlation function $\left(\psi_{X Y}(i)\right)$ between two consecutive CC sets $X$ and $Y$ is defined in (9) as the sum of all cross-correlation functions between any pair of element sequences in those sets.

$$
\psi_{X Y}(i)=\left\{\begin{array}{ll}
\sum_{n=1}^{N_{C C}} \sum_{n=1}^{K_{C C}-i} w_{n}(k) w_{n+N_{C C}}(k+i)=0, & 0 \leq i \leq K_{C C}-1 \\
\sum_{n=1}^{N_{C C}} \sum_{k=1}^{K_{C C}+i} w_{n}(k) w_{n+N_{C C}}(k-i)=0, & 1-K_{C C} \leq i \leq-1
\end{array}\right\}
$$

From (9), it is noted that $\psi_{\boldsymbol{X Y}}(i)$ equals zero for all time shifts, which indicate a high reduction in the MAI between different $\mathrm{CC}$ sequences that belongs to different users. It is assumed that the relative delay between multipaths takes multiple chips. However, if this assumption does not hold it can be shown that the resultant MAI level is far less than that of traditional CDMA systems [10]. It is appropriate to refer to sequence sets which satisfy (8) and (9) as orthogonal CC sequences. For example, consider $\boldsymbol{X}=\left[w_{1}, w_{2}\right]=$ $[1,1,1,-1 ; 1,-1,1,1] \quad$ and $\quad \boldsymbol{Y}=\left[w_{3}, w_{4}\right]=[1,1,-1,1$; $-1,-1,-1,-1]$. Hence, $\psi_{X X}=\psi_{w_{1} w_{1}}+\psi_{w_{2} w_{2}}=[0,0,0$, $8,0,0,0] \quad \psi_{Y Y}=\psi_{w_{3} w_{3}}+\psi_{w_{4} w_{4}}=[0,0,0,8,0,0,0] \quad$ and $\psi_{X Y}=\psi_{w_{1} w_{3}}+\psi_{w_{2} w_{4}}=[0,0,0,0,0,0,0]$.

Comparing (8) and (9) to the three possible values $-t(n)$, $-1, t(n)-2$ of the auto-correlation and cross-correlation functions, indicated in [15], for a set of $N+1$ Gold sequences [16] of length $N=2^{n}-1$. These values are given by

$$
t(n)= \begin{cases}1+2^{\frac{n+1}{2}} & \text { for } n \text { odd } \\ 1+2^{\frac{n+2}{2}} & \text { for } n \text { even }\end{cases}
$$

From above comparison, one can expect the performance of a CC-CDMA system to surpass that of GoldCDMA. This observation agrees with results obtained in [10]. Other sequences, which are of great interest in synchronous spread spectrum networks, are the $\mathrm{m}$-sequences, since the correlation value between different shifts for each m-sequence is almost zero. However, the m-sequences may still be prone to large crosscorrelation values. Walsh obtained the following lower bound on the cross-correlation values between any pair of binary sequences of period $T$ in a set of $M$ sequences [15]

$$
R_{c}(k) \geq T \sqrt{\frac{M-1}{M T-1}} \cong \sqrt{T}
$$


- Since each user in the proposed CDMA system is assigned a signature sequence comprising a set of element sequences, as shown in Table 2, the processing gain of the $\mathrm{CC}$ sequences equals to the aggregate length of all element sequences in a set and can be given by

$$
P G_{C C}=K_{C C} N_{C C}
$$

Those element sequences can be sent to the receiver either using parallel multi-carrier (MC) transmission mode, where all element sequences are transmitted using different carriers or using serial transmission mode, where all element sequences are sent using the same carrier. In this paper, a synchronous serial-transmission mode is adopted.

\section{MIMO CC-CDMA system model}

Figure 1 shows a block diagram of the proposed $(M, P)$ MIMO CC-CDMA system model, where $M$ and $P$ represent the number of transmit and receive antennas. At the transmitter, information bits are de-multiplexed into $M$ parallel streams. Each stream is modulated then further de-multiplexed into $F$ parallel sub-streams, as shown in (13), before being spread by $F$ sets of CC sequences.

$$
\boldsymbol{B}=\left[\begin{array}{ccc}
b_{(1,1,1)} & \ldots & b_{\left(1, F, K_{C C}\right)} \\
\vdots & \ddots & \vdots \\
b_{(M, 1,1)} & \ldots & b_{\left(M, F, K_{C C}\right)}
\end{array}\right]
$$

where $b_{(m, f, j)}$ represents the $j$ th symbol, $\left(j=1,2, \ldots, K_{C C}\right)$ that will be spread using the element sequences of the $f$ th set. Finally the spread signals are summed and normalised, with respect to the signal energy, before being simultaneously transmitted over $M$ antennas. The transmitted signal over the $m$ th antenna is given by

$$
\begin{aligned}
\boldsymbol{t}_{m}= & \left\{\sum_{f=1}^{F} \sum_{j=1}^{K_{C C}} b_{(m, f, j)} \times w_{((f-1) \times F+1)}\left(t-(j-1) \times \tau_{c}\right), \ldots,\right. \\
& \left.\sum_{f=1}^{F} \sum_{j=1}^{K_{C C}} b_{(m, f, j)} \times w_{(f \times F)}\left(t-(j-1) \times \tau_{c}\right)\right\}
\end{aligned}
$$

The multipath channel impulse response is given by

$$
h(t)=\sum_{p=1}^{P} \sum_{l=1}^{L} \sum_{m=1}^{M} h_{(p, l, m)}(t) \delta\left(t-l \tau_{C}\right)
$$

where $h_{p, l, m}$ is the channel coefficient, with zero mean and Rayleigh fading distribution, for the sub-channel between the $p$ th receive antenna and $m$ th transmit antenna over the $l$ th multipath (where $l=1,2, \ldots, L$ ). The received signal at the $p$ th antenna is given by

$$
\boldsymbol{r}_{p}=\sum_{m=1}^{M} \sum_{l=1}^{L} \boldsymbol{t}_{m}\left(t-(l-1) \times \tau_{C}\right) \times h_{(p, l, m)}+\boldsymbol{n}_{p}
$$

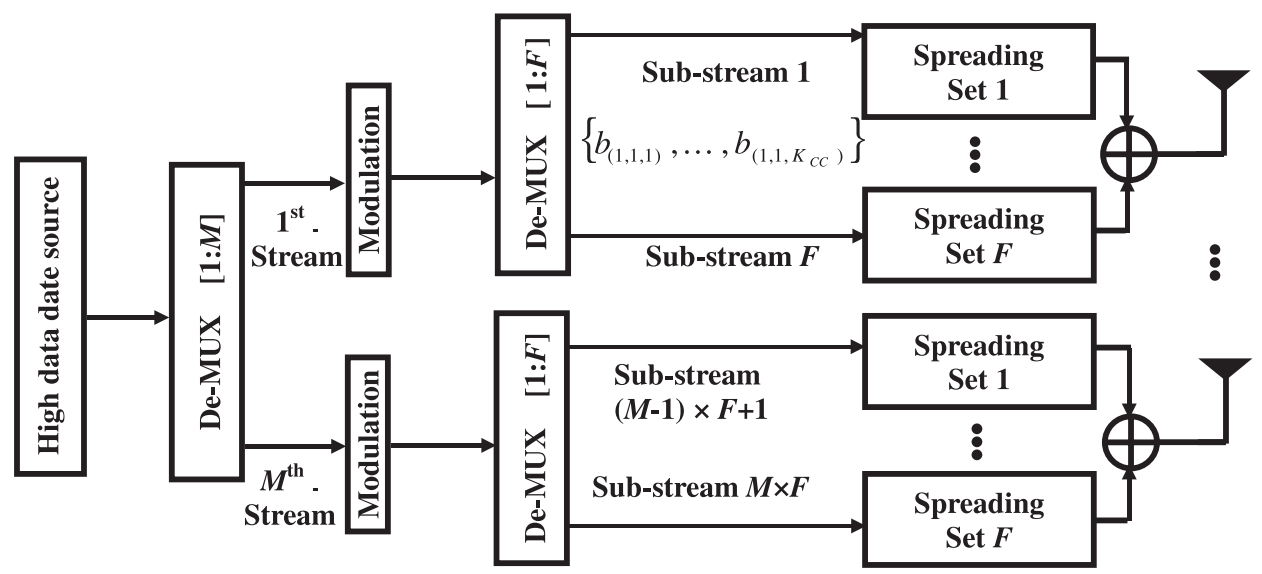

a

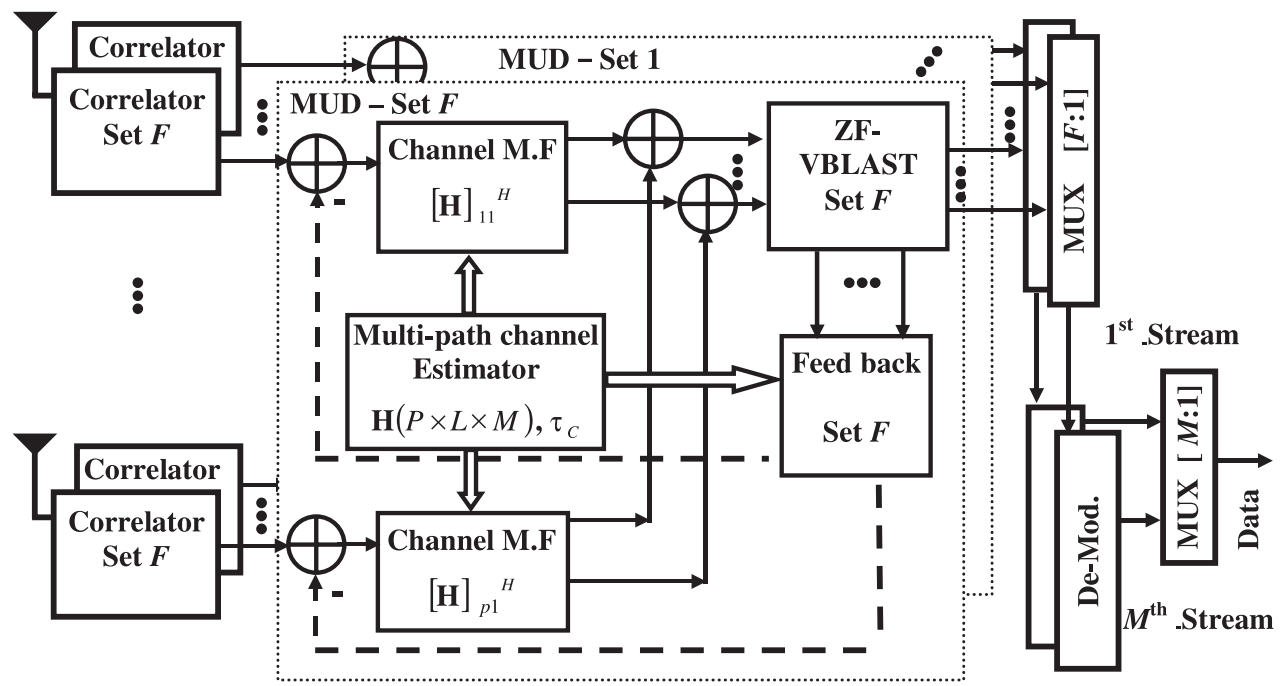

$b$

Fig. 1 Block diagram of the proposed (M,P) MIMO CC-CDMA system model with F sets of CC sequences 
$\boldsymbol{n}_{p}$ represents AWGN with zero mean and variance $\sigma^{2}$. Note that for the simplicity of the following derived equations, the noise power $\sigma^{2}$ is assumed to be zero.

At the receiver, signals at each antenna are de-spread using $F$ set correlators then normalised with $P G_{C C}$. Consider an $(F \times P)$ matrix $\boldsymbol{G}$

$$
\begin{aligned}
{[\boldsymbol{G}]_{f p} } & =\boldsymbol{g}_{f p}^{(j)} \\
& =\left\{\begin{array}{lc}
\sum_{m=1}^{M} b_{(m, f, j)} \times h_{(p, j, m)} & j=1 \\
\sum_{l=1}^{L} \sum_{m=1}^{M} b_{(m, f,(j-l)+1)} \times h_{(p, l, m)} & 2 \leq j \leq K_{C C}
\end{array}\right.
\end{aligned}
$$

where $\boldsymbol{g}_{f p}$ represent the signal at the output of the $f$ th set's correlator that belongs to the $p$ th branch of the receiver. $\boldsymbol{g}_{f p}$ is a single dimensional matrix (dimensions $\left(1 \times K_{C C}\right)$ ), and $\boldsymbol{g}_{f p}(j=1)$ gives the 1st element of matrix $\boldsymbol{g}_{f p}$. The resulting signals in (17) are then passed to $F$ MUDs, each consisting of the following. (i) A channel power profile estimator; including multipath delays and power ratios. (ii) $P$ channel matched filters. The input signals for the $p$ th filter are multiplied by the complex conjugate of the 1st path $(l=1)$ channel coefficients at the $p$ th receive antenna and are given in the following

$$
[\boldsymbol{H}]_{p l}=\boldsymbol{h}_{p l}=\left[h_{p 11}, \ldots, h_{p 1 m}\right]^{T}
$$

where $\boldsymbol{H}$ is the $(P \times L \times M)$ dimensions multipath channel matrix with elements $h_{p, l, m} . T$ stands for the transpose. (iii) A ZF VBLAST detector to eliminate SPI between users sharing the same set of CC sequences and transmitted from different antennas. (iv) A feedback stage, shown in Figs. 1 and 2, that utilises a successive interference canceller (SIC) to eliminate ISI between the data symbols of signals, which are received over different paths in the channel and belong to the same user.

To explain the iterative operation of each MUD a detailed example of $(2,2)$ antenna CC-CDMA system with $K_{C C}=4$ chips and $F=2$ sets, is illustrated in Figs. 3 and 4 , using the block diagram of the receiver and the flowchart of MUD operation, respectively.
At the 1st iteration $(j=1)$ and from (17) and (18) the following is obtained

$$
[\boldsymbol{X}]_{f p}=\boldsymbol{x}_{f p}^{(j)}=\boldsymbol{h}_{p l}^{*} \times \sum_{m=1}^{M} b_{(m, f, j)} \times h_{(p, 1, m)}
$$

where $\boldsymbol{x}_{f p}$ is the output of the $f$ th set weighted by the $p$ th channel matrix that corresponds to the $p$ th receive antenna. The multipath components of the 1st set which form the inputs of the 1st ZF-VBLAST detector [4] are given by

$$
[\boldsymbol{Y}]_{f m}=\boldsymbol{y}_{f m}=\sum_{p=1}^{P} \boldsymbol{x}_{f p}^{(m)}
$$

where $\boldsymbol{y}_{f m}$ is the $m$ th input of the $f$ th ZF-VBLAST. The VBLAST algorithm requires the $M$-by- $M$ set-channel correlation matrix defined as

$$
\boldsymbol{R}_{f}=\sum_{p=1}^{P} \boldsymbol{H}_{p}^{H} \boldsymbol{S}_{f} \boldsymbol{H}_{p}
$$

where $H$ is a Hermitian symmetric matrix and $\boldsymbol{S}_{f}$ is the $L$-by- $L$ set correlation matrix for the $f$ th set. The $(i, j)$ th element of $\boldsymbol{S}_{f}$ is the inner product of the $i$ chip-delayed set with that of the $j$ chip-delayed set. For example, assume $L=2$ multipaths, with the delay of the $l=1$ multipath relative to the $l=2$ multipaths is a single chip, then and from (8) and (9)

$$
\boldsymbol{S}_{f}=\left[\begin{array}{ll}
\psi_{f f}(0) & \psi_{f f}(1) \\
\psi_{f f}(1) & \psi_{f f}(0)
\end{array}\right]=\boldsymbol{I}_{(2 \times 2)}
$$

Equation (21) indicates that by using $\mathrm{CC}$ sequences (which have ideal correlation properties) $\boldsymbol{S}_{f}$ is simplified to an identity matrix, which in turn reduces the complexity of calculating $\boldsymbol{R}_{f}$ as in (21). The reduction of $\boldsymbol{S}_{f}$ into an identity matrix also translates into cancellation of multipath interference, which implies that the VBLAST detectors now operate under flat fading channel conditions. This results in performance achieving a large fraction of the MIMO channel capacity [17].

The channel correlation matrix used in the ZF-VBLAST detector transfer the channel condition from frequency selective to flat fading, hence, the performance of

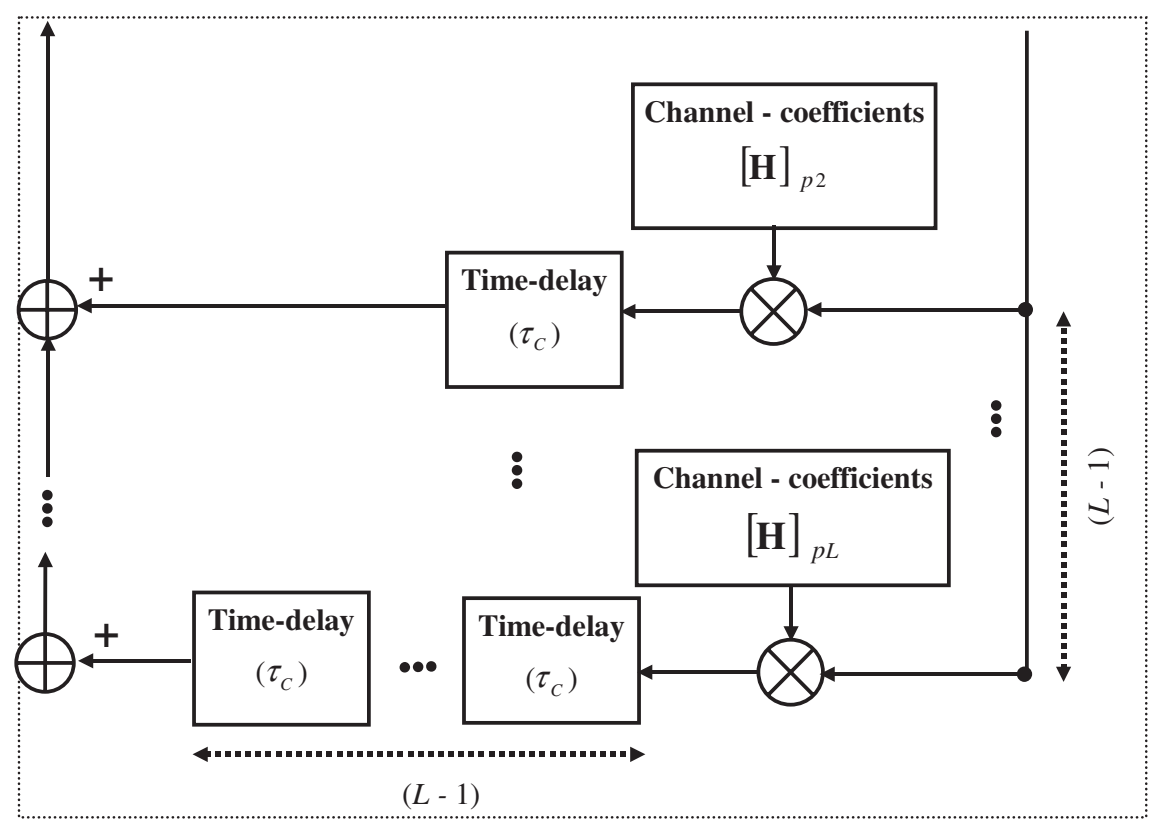

Fig. 2 Block diagram of the feedback stage of the fth set of CC sequences for a multipath channel with L paths 


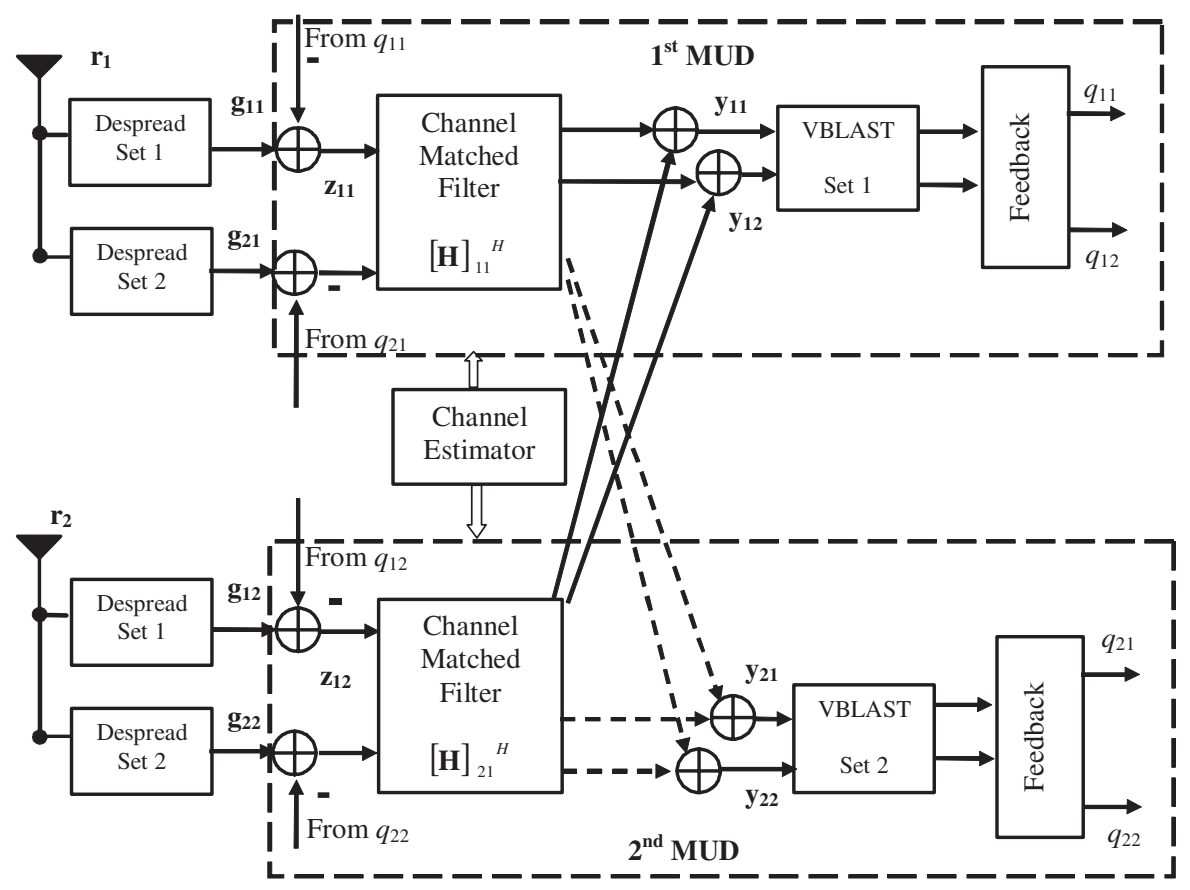

Fig. 3 Receiver block diagram for a $(2,2)$ MIMO CC-CDMA system

CC-CDMA will approximate that of Walsh-CDMA in flat fading as shown in Fig. 5. Referring to (19)-(21) yields

$$
\boldsymbol{Y}=\boldsymbol{R}_{f} \cdot \boldsymbol{b}_{f}^{(j)}
$$

where $\boldsymbol{b}_{f}^{(j)}$ gives the estimated data symbols at the outputs of the $f$ th VBLAST detector at the jth iteration. $\boldsymbol{b}_{f}^{(j)}$ is then scaled by the channel coefficients of the 2 nd path as shown in (25).

$$
\begin{gathered}
{[\boldsymbol{H}]_{12}=\boldsymbol{h}_{12}=\left[\begin{array}{ll}
h_{121} & h_{12 m}
\end{array}\right]} \\
{[\boldsymbol{Q}]_{f p}=\boldsymbol{q}_{f p}^{(j)}=\boldsymbol{h}_{p l} \times \sum_{m=1}^{M} b_{(m, f, j)} \times h_{(p, 1, m)}}
\end{gathered}
$$

In order to eliminate ISI of the next detection stages, signals in (25) are subtracted from those in (17) at the $(j+1)$ th iteration to give the following

$$
[\boldsymbol{Z}]_{f p}=\boldsymbol{z}_{f p}^{(j+1)}=\boldsymbol{g}_{f p}^{(j+1)}-\boldsymbol{q}_{f p}^{(j+1)}
$$

The iterative operation of the MUD is repeated for $K_{C C}$ iterations.

\section{$4 \quad$ Simulation models and numerical results}

Table 3 provides the simulation parameters for comparable $(2,2)$ CC-CDMA and Walsh-CDMA system models. The multipath channel model is set up with the following assumptions and parameters: (i) a frequency-selective Rayleigh fading distribution [18] and uncorrelated fading between the antenna pairs; (ii) perfect channel estimation; (iii) each sub-channel has $L=2$ components with the 2 nd path delayed by $\tau_{C}$. The average-power ratio between the two paths is defined as

$$
\begin{aligned}
& \text { channel-power-ratio (CPR) } \\
& \quad=(2 \text { nd path power } / 1 \text { st path power })
\end{aligned}
$$

The simulations assume an ideal modulation and demodulation process leaving the effects of multilevel carrier modulation and demodulation as a topic of future study.

Results shown in Fig. 5 are twofold: first, although the BER performance of Walsh-CDMA out performs that of

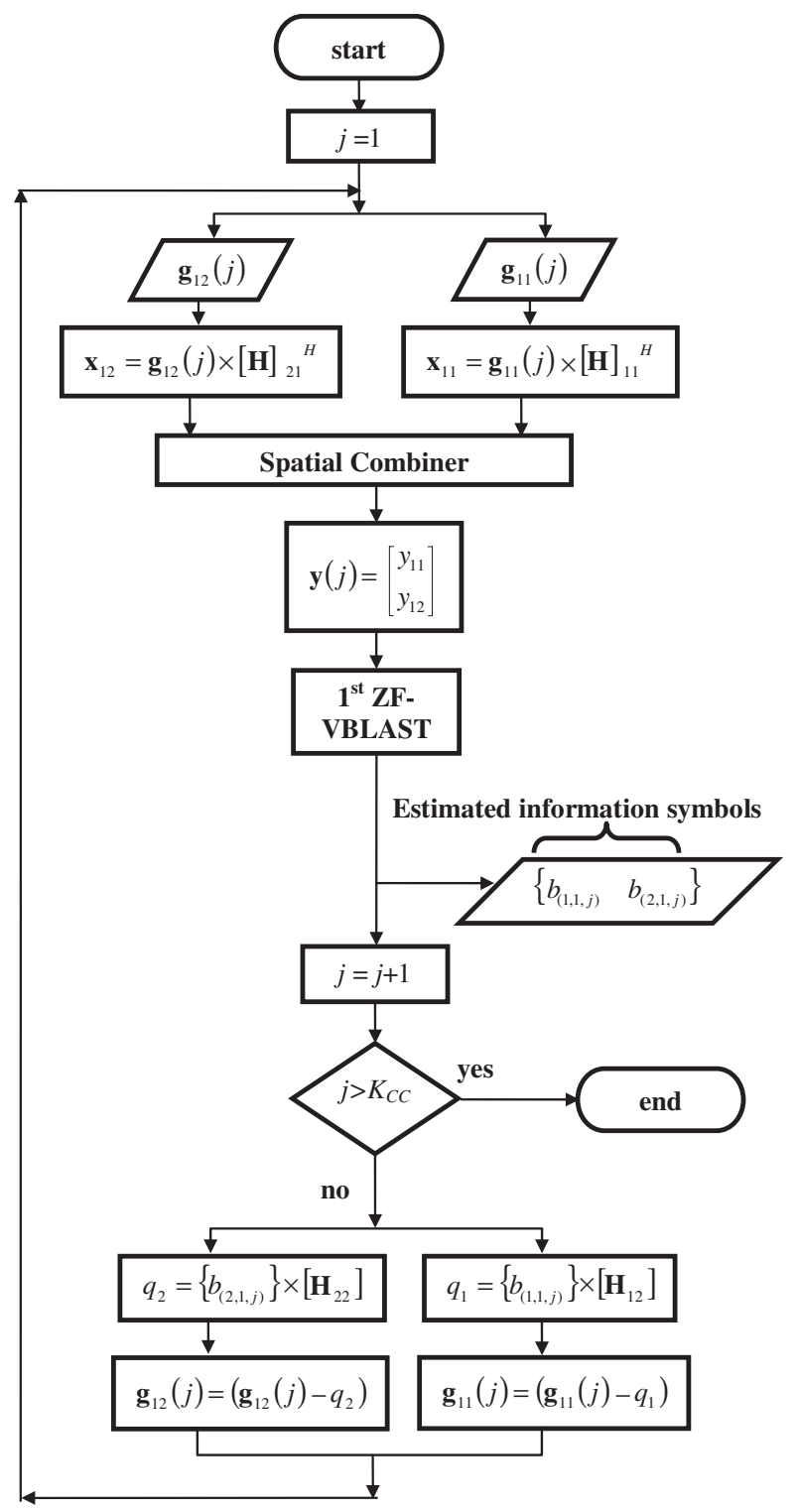

Fig. 4 Flow chart of the 1st MUD that estimates symbols spread by the 1st set of CC sequences 


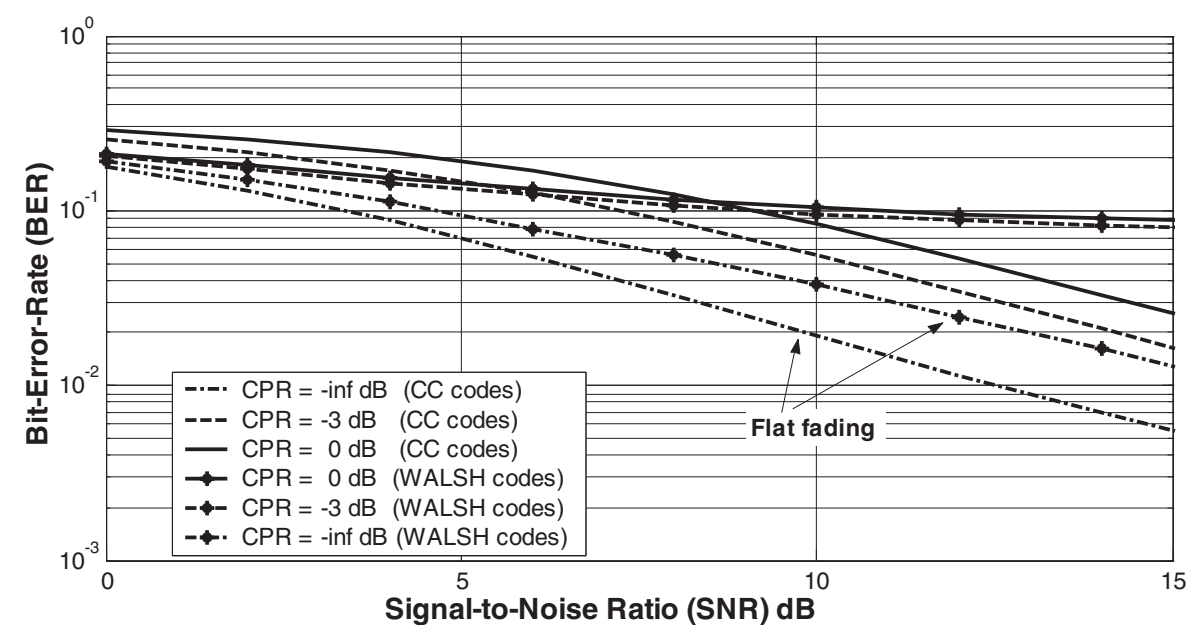

Fig. 5 BER against SNR for (2, 2) Walsh-CDMA and CC-CDMA systems with similar user capacity and processing gain

CC-CDMA, for CPR $=0 \mathrm{~dB}$ when $\mathrm{SNR}$ is under $8 \mathrm{~dB}$ and for $\mathrm{CPR}=-3 \mathrm{~dB}$ when $\mathrm{SNR}$ is under $6 \mathrm{~dB}, \mathrm{CC}-\mathrm{CDMA}$ illustrates a dramatic improvement in performance over the Walsh-CDMA system to such an extent that the CCCDMA performance at $\mathrm{CPR}=0 \mathrm{~dB}$ has only a mere $3 \mathrm{~dB}$ degradation from the Walsh-CDMA operating at a $\mathrm{CPR}=-$ infinity $\mathrm{dB}$. Second, although a drop in performance $(\approx 6 \mathrm{~dB})$ for a CC-CDMA system operating at $\mathrm{CPR}=0 \mathrm{~dB}$ compared to the case for flat fading channels, no IBER is present for any of the CPR values owing to the novel combination of CC sequences and the MUD, proposed in this paper, which effectively mitigate the effect of MAI, ISI and SPI sources.

Table 3: Simulation parameters of both MIMO CC-CDMA and MIMO Walsh-CDMA systems

\begin{tabular}{lll}
\hline Simulation parameters & CC-CDMA & Walsh-CDMA \\
\hline Element sequence length & $K_{C C}=4$ & $K_{W}=8$ \\
Processing gain & $P G_{C C}=8$ & $P G_{W}=8$ \\
$\begin{array}{l}\text { Number of users supported } \\
\text { Sub-streams data rate }\end{array}$ & 4 & $4(50 \%$ system load) \\
$\begin{array}{l}\text { (ksymbol/s) } \\
\text { Transmit chipping rate }\end{array}$ & 3.84 & $S R_{W C}=960$ \\
$\begin{array}{l}\text { (Mchip/s) } \\
\text { Total data rate (Mbit/s) }\end{array}$ & 7.68 & 3.84 \\
\hline
\end{tabular}

The degradation in CC-CDMA performance is due to the use of a simple low-complexity single-looped recursive filter, at the feedback stage shown in Fig. 2, which while trying to cancel the ISI, between received data sub-streams caused by the time-dispersive effect of a frequency-selective fading channel, it does not cancel the noise. This results in the error propagation problem associated with the SIC algorithm [19]. Note that results in Fig. 5 are consistent with those obtained in [10].

A more advanced SIC is likely to result in less degradation in system performance for low SNR.

Figure 6 illustrates a comparison in BER performance for CC-CDMA using $K_{C C}=4$, and 16. The results obtained are threefold: first, approximately similar flat fading $(\mathrm{CPR}=-$ infinity $\mathrm{dB})$ performance; second, the resilience of CC-CDMA systems to an increased number of users $\left(K_{C C}=16\right)$, when operating in frequency selective fading channels $(\mathrm{CPR}=0 \mathrm{~dB})$, with $\approx 1 \mathrm{~dB}$ degradation compared with $K_{C C}=4$; finally, the results highlight the possibility of creating a multi-rate and multi-user system that offers variable HDR services without compromising the acceptable error rate levels in the system [20].

\section{Conclusions}

In this paper a novel and practical MIMO CDMA architecture based on $\mathrm{CC}$ sequences has been proposed and simulated for different numbers of users and channel profile using BER as a measure of system performance. The

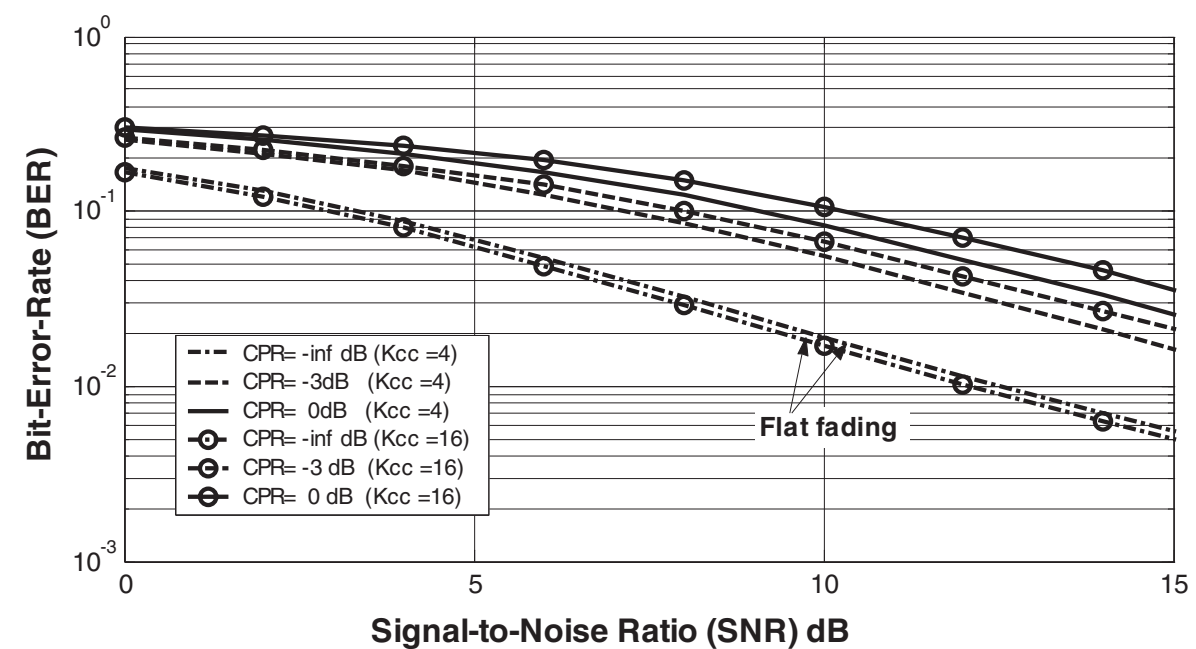

Fig. 6 BER against $S N R$ for a $(2,2) C C-C D M A$ system with $K_{C C}=4$, and 16 
results achieved are twofold. First, the proposed MIMO CC-CDMA system overcomes the problem associated with the low user-capacity of SISO CC sequences, by using the 'set re-use' technique that supports multi-user transmission mode of up to $M \times F$ users in a MIMO antenna, or $M$ times higher total data rate compared to a SISO CCCDMA system. Secondly, the MIMO CC-CDMA system significantly outperforms the traditional MIMO WalshCDMA systems in a frequency-selective fading environment with BER performance of MIMO CC-CDMA in frequency selective fading approaching that of MIMO Walsh-CDMA in flat fading channel conditions. Overall this work highlights the significant opportunities that are presented to designers of MIMO systems by utilising the inherent properties of $\mathrm{CC}$ sequences.

\section{References}

1 Foschini, J.G., and Gans, J.M.: 'On limits of wireless communications in a fading environment when using multiple antennas', Wirel. Pers. Commun., 1998, 6, (3), pp. 311-335

2 Goldsmith, A., Jafar, A.S., Jindal, N., and Vishwanath, S.: 'Capacity limits of MIMO channels', IEEE J. Sel. Areas Commun., 2003, 21, (5), pp. 684-702

3 Paulraj, A., Nabar, R., and Gore, D.: 'Introduction to space-time wireless communications' (Cambridge University Press, 2003)

4 Golden, G.D., Foschini, J.G., Valenzuela, A.R., and Wolniansky, W.P.: 'Detection algorithm and initial laboratory results using V-BLAST space-time communication architecture', Electron. Lett., 1999, 35, (1), pp. 14-16

5 Huang, H., Viswanathan, H., Blanksby, A., and Haleem, A.M.: 'Multiple antenna enhancements for a high rate CDMA packet data system', J. VLSI Signal Process. Syst. Signal Image Video Technol., 2002, 30, pp. 55-69

6 Boubaker, N., Letaief, B.K., and Murch, D.R.: 'Performance of BLAST over frequency-selective wireless communication channels', IEEE Trans. Commun., 2002, 50, (2), pp. 196-199
7 Leus, G., Petre, F., and Moonen, M.: 'Space-time chip equalization for space-time coded downlink CDMA'. Proc. IEEE Int. Conf. on Communications (ICC'02), New York, USA, 28 April-2 May 2002, pp. $568-572$

8 Ng, W.K, Cheng, S.R., and Murch, R.D.: 'A simplified bit allocation for V-BLAST based OFDM MIMO systems in frequency selective fading channel'. Proc. IEEE Int. Conf. on Communications (ICC'02), New York, USA, 28 April-2 May 2002, pp. 411-415

9 Li, J., and Kavehrad, M.: 'Effects of Time Selective multipath fading on OFDM systems for broadband mobile Applications', IEEE Commun. Lett., 1999, 3, (12), pp. 332-334

10 Chen, H.H., Yeh, F.J., and Suehiro, N.: 'A multicarrier CDMA architecture based on orthogonal complementary codes for new generations of wideband wireless communications', IEEE Commun. Mag., 2001, 39, (10), pp. 126-135

11 Popovic, M.B.: 'Spreading sequences for multicarrier CDMA systems', IEEE Trans. Commun., 1999, 47, (6), pp. 918-926

12 Turyn, R.: 'Ambiguity function of complementary sequences', IEEE Trans. Inf. Theory, 1963, IT-9, pp. $46-47$

13 Taki, Y., Miyakawa, H., Hatori, M., and Namba, S.: 'Even-shift orthogonal sequences', IEEE Trans. Inf. Theory., 1969, IT-15, pp. 295-300

14 Suehiro, N., and Hatori, M.: 'N-shift cross-orthogonal sequences', IEEE Trans. Inf. Theory, 1988, IT-34, pp. 143-46

15 Dinan, H.E and Jabbari, B.: 'Spreading codes for direct sequence CDMA and wideband cdma cellular networks', IEEE Commun. Mag., 1999, 36, (9), pp. 48-54

16 Gold, R.: 'Optimal binary sequences for spread spectrum multiplexing', IEEE Trans. Inf. Theory, 1967, IT-13, pp. 619-21

17 Foschini, G., Golden, G., Valenzuela, R.A., and Wolniansky, W.P. 'Simplified processing for wireless communications at high spectral efficiency', IEEE J. Sel. Areas Commun., 1999, 17, pp. 1841-1852

18 Dent, P., Bottomley, G.E., and Croft, T.: 'Jakes fading model revisited', Electron. Lett., 1993, 29, (13), pp. 1162-1163

19 Shen, C., Zhuang, H., Dai, L., and Zhou, S.: 'Detection algorithm improving V-BLAST performance over error propagation', Electron. Lett., 2003, 39, (13), pp. 1007-1008

20 Horng, H., and Derand, T.: 'Interference cancellation in CDMA downlink supporting variable rate services'. IEEE Int. Conf. on Consumer Electronics (ICCE'01), Los Angeles, CA, USA, 19-21 June 2001, pp. 100-101 